\title{
ARTICLE
}

\section{A previously unrecognised phenotype characterised by obesity, muscular hypotonia, and ability to speak in patients with Angelman syndrome caused by an imprinting defect}

\author{
Gabriele Gillessen-Kaesbach ${ }^{1}$, Stephanie Demuth ${ }^{2}$, Hannelore Thiele ${ }^{3}$, Ursel Theile ${ }^{4}$, \\ Christina Lich $^{1}$ and Bernhard Horsthemke ${ }^{1}$ \\ ${ }^{1}$ Institut für Humangenetik, Universitätsklinikum Essen \\ ${ }^{2}$ Praxis für Humangenetische Beratung, Erfurt \\ ${ }^{3}$ Institut für Humangenetik und Medizinische Biologie der Universität Halle \\ ${ }^{4}$ Genetische Beratungstelle des Landes Rheinland-Pfalz, Mainz, Germany
}

\begin{abstract}
The clinical features of Angelman syndrome (AS) comprise severe mental retardation, postnatal microcephaly, macrostomia and prognathia, absence of speech, ataxia, and a happy disposition. We report on seven patients who lack most of these features, but presented with obesity, muscular hypotonia and mild mental retardation. Based on the latter findings, the patients were initially suspected of having Prader-Willi syndrome. DNA methylation analysis of $S N R P N$ and D15S63, however, revealed an AS pattern, ie the maternal band was faint or absent. Cytogenetic studies and microsatellite analysis demonstrated apparently normal chromosomes 15 of biparental inheritance. We conclude that these patients have an imprinting defect and a previously unrecognised form of AS. The mild phenotype may be explained by an incomplete imprinting defect or by cellular mosaicism.
\end{abstract}

Keywords: Angelman syndrome; imprinting defect

\section{Introduction}

Angelman syndrome (AS) and Prader-Willi syndrome (PWS) are distinct genetic disorders caused by a deficiency of imprinted gene expression from the maternal or paternal chromosome 15. AS is characterised by microcephaly, severe mental retardation, lack of speech, macrostomia and prognathia, ataxia, seizures, and a happy disposition. ${ }^{1-3}$ In infants and

Correspondence: Gabriele Gillessen-Kaesbach MD, Institut für Humangenetik, Universitätsklinikum Essen, Hufelandstr 55, D-45122 Essen, Germany. Tel: (+ +49) 201723 4563; Fax: (++49) 201723 5900; E-mail: g.gillessen@uni-essen.de

Received 29 January 1999; revised 13 April 1999; accepted 20 April 1999 adults the clinical diagnosis is often difficult and therefore the frequency of AS is probably underestimated. PWS is characterised by muscular hypotonia and feeding problems in newborns, obesity, short stature, hypogonadism and behavioural problems. Mental retardation in PWS is usually much milder than in AS. PWS is often wrongly suspected in patients with obesity and mental retardation, which occur in many different disorders. Today, the clinical diagnosis of PWS can easily be confirmed by molecular tests. ${ }^{4}$ In contrast, $20 \%$ of AS patients have no detectable molecular defect.

Several molecular defects leading to the loss of function of the $A S$ gene(s) and $P W S$ gene(s) have been 
recognised. ${ }^{5}$ Approximately $70 \%$ of patients with AS and PWS have a de novo maternally or paternally derived deletion in the chromosomal region 15q11-q13. Most of the other patients with PWS have two chromosomes 15 of maternal origin (uniparental maternal disomy), whereas uniparental paternal disomy occurs in only $1-2 \%$ of patients with AS. Mutations of the $U B E 3 A$ gene, which is imprinted in brain cells, are found in $2-4 \%$ of AS patients. A small number of patients with PWS and AS have apparently normal chromosomes of biparental origin, but the paternal chromosome 15 carries a maternal imprint (PWS) or the maternal chromosome carries a paternal imprint (AS). Such an imprinting defect can result from an imprinting centre (IC) mutation or occur spontaneously. ${ }^{6,7,8}$

Genotype-phenotype correlations in PWS and AS have revealed subtle clinical differences. ${ }^{5}$ It appears as if microcephaly is less frequent in AS patients with an imprinting defect. ${ }^{8}$ Furthermore, there are some case reports about AS patients with uniparental disomy and a milder phenotype. ${ }^{9-12}$ It should be noted, however, that genotype-phenotype studies can suffer from an ascertainment bias, because in general the patients are diagnosed according to standard diagnostic criteria., ${ }^{2,3}$ Thus, patients with atypical or very mild phenotypes may usually not be included in such studies and most often are identified by chance only. Here we report on a series of seven AS patients, who presented with some features of PWS. Interestingly, all of these patients have an imprinting defect. Our findings extend the pre- viously known phenotypic spectrum of AS and may help to recognise more such patients.

\section{Subjects and Methods}

\section{Ascertainment}

Between 1993 and 1998 our diagnostic laboratory received blood samples from six patients who were suspected of having PWS, but turned out to have an AS methylation pattern. Two of these patients were then examined personally by one of the authors (GG-K). In another case, the mother suspected her daughter of having PWS and presented the child to this author.

\section{Case Reports}

Clinical pictures of patients $1-3,5$ and 7 are depicted in Figure 1 and Figure 2 .

Patient 1 (LM12690) Patient 1 was born as the second son to healthy parents. His birth weight was $4050 \mathrm{~g}(+1 \mathrm{SD})$, length $54 \mathrm{~cm}$ (mean) and OFC $36.5(+1 \mathrm{SD})$. In spite of muscular hypotonia, his motor development was normal as he started walking independently at 16 months. A striking appetite was noted from the first months of life. At the age of 3 years his height was of $93 \mathrm{~cm}(-1.5 \mathrm{SD})$ and he weighed $18 \mathrm{~kg}$ $(+3 \mathrm{SD})$. At age 7 years 5 months his height is $124 \mathrm{~cm}$ $(-0.5 \mathrm{SD})$ and his weight $32 \mathrm{~kg}(+2.5 \mathrm{SD})$. He has a happy disposition and speaks a few words; his comprehension is very good. He has neither ataxia nor epilepsy. PWS was diagnosed clinically when he was 3 years old. This patient's data were partly described by Bürger et al.

Patient 2 (BA13394) This girl was born to healthy parents after an uneventful pregnancy with a birth weight of $4850 \mathrm{~g}$ $(+4 \mathrm{SD})$, length of $58 \mathrm{~cm}(+3 \mathrm{SD})$ and an OFC of $38 \mathrm{~cm}$ (+3SD). Feeding problems were noted in the newborn period, but no severe neonatal muscular hypotonia. She
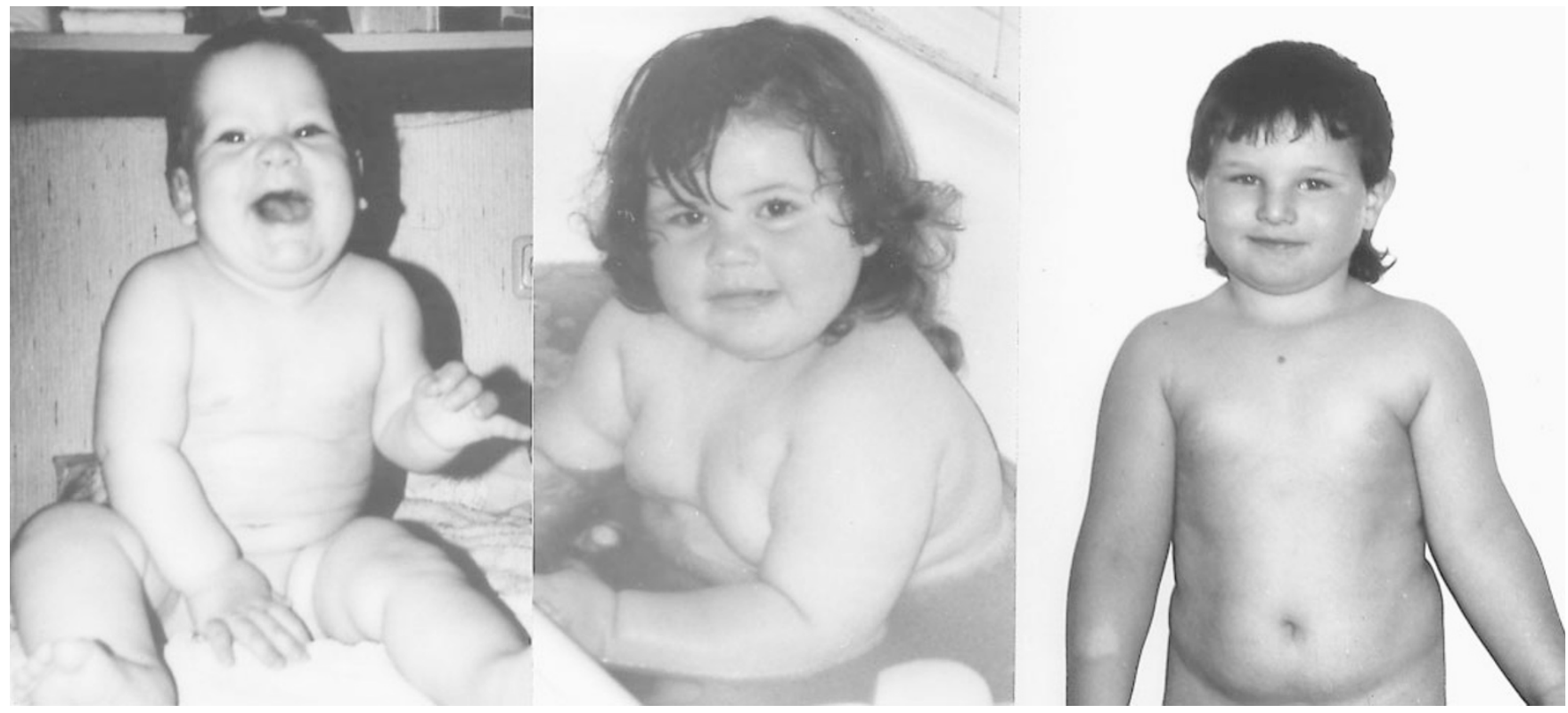

Figure 1 Clinical photographs of atypical patients with AS syndrome. Note obesity (Patients 1-3) 
walked unaided at $2 \frac{1}{2}$ years of age. So far at age 3 years and 5 months, no active speech is present, although comprehension is described as good. The parents report sleeping problems. She developed obesity when about 12 months old. She is considerably macrosomic with a weight of $35 \mathrm{~kg}$ (+5SD) and a height of $108 \mathrm{~cm}(+2 \mathrm{SD})$. Her OFC is $54 \mathrm{~cm}$ (+3.5SD). She has neither ataxia nor epilepsy. Facial features are normal and not suggestive of AS. Neuropaediatric examination was performed to identify the etiology of her developmental delay and obesity, and a clinical diagnosis of PWS was suggested.

Patient 3 (HS311289) Patient 3 was delivered by Caesarean section as the second child to non-consanguineous parents. Birth weight was $3800 \mathrm{~g}$ (mean) and length $50 \mathrm{~cm}$ $(-1.5 \mathrm{SD})$. His older sister had a ventricular septal defect. From his first year of life developmental delay and muscular hypotonia have been obvious. He walked unsupported at $2 \frac{1}{2}$ years of age. Speech development is severely impaired. At his present age of 8 years he is able to speak only two or three words. He developed seizures at the age of 12 months requiring antiepileptic therapy. Obesity has been present from early on. His height corresponds to the 25th percentile for age, OFC to P25, and his weight is above the 97th percentile. He presents with muscular hypotonia, micropenis and behaviour problems. Ataxia is not present. Facial features include almond-shaped eyes, downturned corners of the mouth and hypoplastic alae nasi. A clinical geneticist tentatively classified his clinical features as PWS.

Patient 4 (DS25393) This girl was born after 36 weeks of gestation with a birth weight of $3220 \mathrm{~g}(+1 \mathrm{SD})$, a length of $53 \mathrm{~cm}(+1.5 \mathrm{SD})$ and a head circumference of $35 \mathrm{~cm}$ $(+1.5 \mathrm{SD})$. The parents were healthy and non-consanguineous. She presented with muscular hypotonia and her developmental milestones were delayed: at the age of 2 years she started to walk unaided and could say 'mama' and 'papa'. She has been overweight from her first months of life. During infancy she had a total of five febrile seizures. The awake EEG pattern shows a slight diminished activity. Rarely there are diffuse high-amplitude $1-3 \mathrm{~Hz}$ slow waves and spikes. She has no epilepsy. We examined her at the age of 5 years and

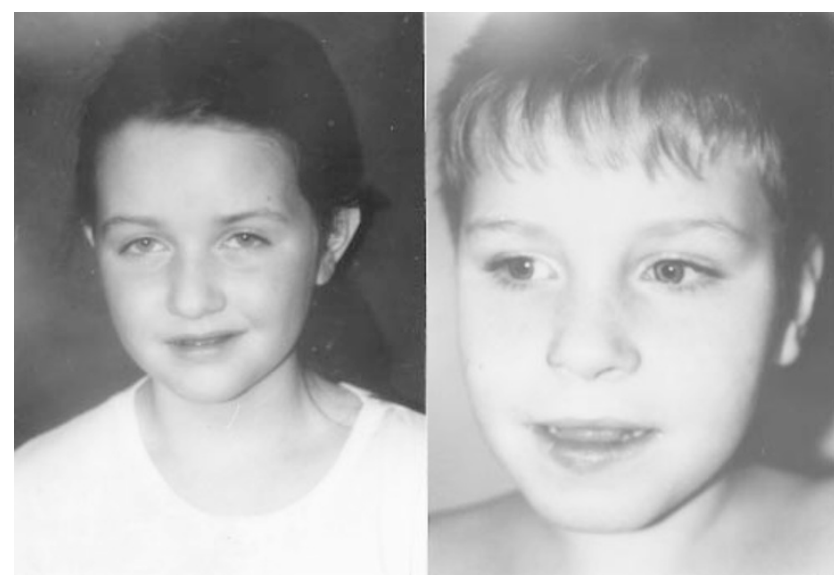

Figure 2 Lack of AS specific facial features (Patients 5 and 7)
6 months. Her height is $114 \mathrm{~cm}$ (mean), weight is $28 \mathrm{~kg}$ (+4SD) and OFC $51 \mathrm{~cm}$ (mean) (Figure 1). She is able to speak about 20 words and comprehension is good. She shows behavioral problems, especially a tendency to be aggressive, and social contact with children of her age is poor. The neurological examination was normal and did not reveal ataxia. The diagnosis of PWS was tentatively made by her paediatrician because of the combination of obesity and speech delay.

Patient 5 (MX29790) This girl was born after an uneventful pregnancy to healthy non-consanguineous German parents as their first daughter. Birth parameters were in the normal range: weight $3000 \mathrm{~g}(-1 \mathrm{SD})$, length $54 \mathrm{~cm}$ (+1SD), OFC 34 (mean). She started walking at 18 months and spoke her first words at the age of 2 years. She now is 7 years 8 months old and is able to talk in two to four word sentences and answers appropriately to questions. Her speech, however, is hypernasal and difficult to understand. She enjoys eating and her weight is $30 \mathrm{~kg}(+1 \mathrm{SD})$. Her height is $129 \mathrm{~cm}$ $(+0.5 \mathrm{SD})$ and $\mathrm{OFC} 50 \mathrm{~cm}(-1 \mathrm{SD})$. She still presents with muscular hypotonia. During her first 6 years of life she had sleeping problems. The awake of EEG pattern shows high amplitude waves. Seizures have not been noticed. The mother knows a girl with PWS and therefore speculated that her daughter might have the same disorder. Being aware of the phenotype of the other patients, one of the authors (GG-K) suspected the girl to belong to a subgroup of patients with AS.

Patient 6 (MJ121095) This boy is the first child of healthy German parents. At term he weighed $3750 \mathrm{~g}$ (mean), length was $54 \mathrm{~cm}$ (mean) and OFC was $36.5 \mathrm{~cm}(+1 \mathrm{SD})$. Breast feeding was difficult and he had to be bottle fed. At the age of 8 months, the mother observed a developmental delay for which he was examined neuropaediatrically. Muscular hypotonia was documented. The EEG revealed delta waves of high amplitude. At our examination at the age of 23 months, he was able to sit unsupported but could not walk unaided. He had no active speech but comprehension was normal for age. His weight was $16 \mathrm{~kg}(+1.5 \mathrm{SD})$ and height $95 \mathrm{~cm}(+2 \mathrm{SD})$. He was macrocephalic with an OFC of $54 \mathrm{~cm}(+3.5 \mathrm{SD})$. The neuropaediatricians suspected he had PWS because of the above mentioned features. At a re-examination at the age of 3.5 years, the patient showed massive obesity $(25 \mathrm{~kg} ;+5 \mathrm{SD})$ His height was $100 \mathrm{~cm}$ (mean). He is able to understand and has started to speak a few words.

Patient 7 (MA311090) This patient is the only child of a young German couple. He was born after 35 weeks of gestation with a birth weight of $2640 \mathrm{~g}$ (mean) and a length of $49 \mathrm{~cm}(+0.5 \mathrm{SD})$. He was reported to have neonatal muscular hypotonia and feeding problems. Obesity was obvious at the age of 4 years. At the age of 7 years and 10 months he had a length of $120 \mathrm{~cm}(-1 \mathrm{SD})$ and a weight of $31 \mathrm{~kg}(+4 \mathrm{SD})$. His OFC was $51 \mathrm{~cm}(-1 \mathrm{SD})$. he is severely retarded with absence of speech, but displays neither ataxia nor epilepsy. Features such as muscular hypotonia, mental retardation and obesity suggested the clinical diagnosis of PWS. Molecular data (patient ASID-16) have been described before by Buiting et $a{ }^{6}$ 


\section{Cytogenetic Analysis and FISH}

Routine cytogenetic analysis including GTG-banding of cultured blood lymphocytes was performed in all patients and their parents according to standard methods. ${ }^{13}$ Fluorescence in situ hybridisation (FISH) was performed in each case (number of metaphases analysed: 50 ) as described by Lichter et $a l^{14}$ using probes specific for the SNRPN and GABRB3 loci, which map to $15 \mathrm{q} 11-\mathrm{q} 13$, and a control probe for the $P M L$ locus, which maps to $15 \mathrm{q} 22$ (ONCOR). The hybridisation and immunochemical detection was carried out according to the manufacturer's recommendations.

\section{DNA Analysis}

DNA was extracted from peripheral blood by standard procedures. The methylation status was analysed by Southern blot analysis with PW71B $(D 15 S 63)^{15}$ and by methylationspecific PCR for $S N R P N{ }^{16}$ To search for microdeletions in the IC, Southern blot hybridisations with probe IC1/3 were performed, which spans the shortest region of deletion overlap in AS patients with an IC deletion. ${ }^{17}$ Microsatellites were studied with fluorescence-labelled primers and standard procedures. $^{6}$

\section{Results}

Between 1993 and 1998 we identified seven patients who were suspected of having PWS, but turned out to have an AS methylation pattern. The patients presented with obesity, muscular hypotonia and mental retardation, but lacked ataxia, microcephaly and facial features typical of AS. Three patients had active speech and were able to communicate with others. The main clinical features are summarised in Table 1.

Southern blot analysis with PW71B (D15S63) revealed a normal paternal band, but a faint maternal band (Figure 3 ). Similar results were obtained by the methylation-specific PCR test for SNRPN: two patients (6 and 7) lacked the maternal band, two patients ( 1 and
3) had a very weak maternal band, and three patients (2, 4 and 5) had a weak maternal band (Figure 3 ).

Cytogenetic analysis at the 400 band stage revealed normal chromosomes in the patients and their parents. FISH analysis with probes for SNRPN and GABRB3 gave signals in 15q11-q13 on both chromosomes (not shown). Thus there was no evidence of a deletion in this region. Microsatellite analysis of the patients and their parents showed biparental inheritance of at least two chromosome 15 loci in each case (Table 2). This result, in combination with the methylation patterns, suggests that the patients have a paternal imprint on the maternal chromosome, ie an imprinting defect. Southern blot analysis with a probe from the imprinting centre region did not reveal any deletion in this region (not shown).

\section{Discussion}

Absence of speech, early onset of seizures, mental retardation, ataxia and postnatal microcephaly represent the major features of Angelman syndrome. ${ }^{1-3}$ Furthermore, the average birth weight in AS patients is $200 \mathrm{~g}$ less than in normal sibs. ${ }^{2}$ Here we report on seven patients who have AS by molecular analysis, but lack most of the typical clinical features. Previous observations focused on a milder phenotype in AS patients with UPD. ${ }^{9-12}$ These patients showed a milder degree of ataxia and mental retardation, normal stature and head circumference as well as obesity. Minassian et $a l^{18}$ reported that AS patients with a typical interstitial deletion of 15q11-q13 have more severe epilepsy than patients with UPD, a $U B E 3 A$ mutation or an imprinting defect. Only one of our patients developed seizures.

Table 1 Clinical findings in 7 AS patients with imprinting defect

\begin{tabular}{|c|c|c|c|c|c|c|c|}
\hline Patients & 1 & 2 & 3 & 4 & 5 & 6 & 7 \\
\hline Weight at birth (g) & 4050 & 4850 & 3800 & $3220^{\mathrm{a}}$ & 3000 & 3750 & $2640^{b}$ \\
\hline Length at birth (cm) & 54 & 58 & 50 & 53 & 54 & 54 & 49 \\
\hline OFC at birth $(\mathrm{cm})$ & 36.5 & 38 & nd & 35 & 34 & 36.5 & nd \\
\hline Muscular hypotonia & + & - & + & + & + & + & + \\
\hline Early onset of obesity & + & + & + & + & + & + & + \\
\hline Ataxia & - & - & - & - & - & - & - \\
\hline Ability to speak & + & - & - & + & + & - & - \\
\hline Epilepsy & - & - & + & - & - & - & - \\
\hline EEG changes & + & - & + & + & + & + & - \\
\hline Hypopigmentation & - & - & - & - & + & - & - \\
\hline Microcephaly & - & - & - & - & - & - & - \\
\hline Mental retardation & + & + & + & + & + & + & + \\
\hline
\end{tabular}

a 36 weeks of gestation; 35 weeks of gestation; nd: not detected; +: present; -: absent. 
Table 2 Segregation analysis of $15 \mathrm{q}$ markers

\begin{tabular}{|c|c|c|c|c|c|c|c|c|c|c|c|c|c|c|c|c|c|c|c|c|c|}
\hline Family & 1 & & & 2 & & & 3 & & & 4 & & & 5 & & & 6 & & & 7 & & \\
\hline Individual & $\mathrm{F}$ & $\mathrm{M}$ & $\mathrm{P}$ & $\mathrm{F}$ & M & $\mathrm{P}$ & $\mathrm{F}$ & M & $\mathrm{P}$ & $\mathrm{F}$ & $\mathrm{M}$ & $\mathrm{P}$ & $\mathrm{F}$ & $\mathrm{M}$ & $\mathrm{P}$ & $\mathrm{F}$ & $\mathrm{M}$ & $\mathrm{P}$ & $\mathrm{F}$ & $\mathrm{M}$ & $\mathrm{P}$ \\
\hline D15S11 & 1010 & 710 & 107 & nd & nd & nd & nd & 11 & 11 & nd & nd & nd & nd & nd & nd & nd & nd & nd & nd & nd & nd \\
\hline D15S817 & nd & nd & nd & 23 & 12 & 21 & nd & 13 & 21 & 31 & 23 & 33 & nd & nd & nd & 23 & 12 & 21 & 12 & 23 & 12 \\
\hline D15S128 & nd & nd & nd & 41 & 33 & 43 & nd & nd & nd & 23 & 13 & 23 & 12 & 34 & 23 & 13 & 22 & 12 & 21 & 13 & 21 \\
\hline D15S63 & 24 & 24 & 24 & nd & nd & nd & nd & nd & nd & nd & nd & nd & nd & nd & nd & nd & nd & nd & nd & nd & nd \\
\hline D15S1234 & nd & nd & nd & 42 & 53 & 45 & nd & 12 & 12 & 21 & 23 & 23 & 23 & 14 & 31 & 63 & 14 & 61 & 31 & 42 & 34 \\
\hline GABRB3 & 310 & 511 & 35 & nd & nd & nd & nd & 11 & 21 & nd & nd & nd & nd & nd & nd & nd & nd & nd & nd & nd & nd \\
\hline CYP19 & nd & nd & nd & 13 & 22 & 12 & nd & nd & nd & 12 & 33 & 13 & 31 & 21 & 12 & 32 & 14 & 31 & 11 & 12 & 11 \\
\hline D15S100 & nd & nd & nd & 22 & 11 & 21 & nd & nd & nd & 11 & 23 & 13 & 12 & 11 & 21 & 12 & 13 & 11 & 31 & 24 & 32 \\
\hline
\end{tabular}

F: father; M: mother; P: patient; nd: not done.

An abnormal EEG pattern was present in $5 / 7$ patients, but this was not consistent with the changes typically seen in AS. In a study describing the phenotypical differences in AS patients of different molecular classes, Bürger et $a l^{8}$ pointed out that microcephaly and hypopigmentation are less frequently observed in patients with imprinting mutations than in patients with deletions. Similar findings have been reported by Moncla et al..$^{5}$ Our study confirms these aspects. ${ }^{5,8,18}$ All patients described by us had a normal head circumference for their age and normal pigmentation. In addition, they had normal or larger birth sizes, and all but one lacked epilepsy.

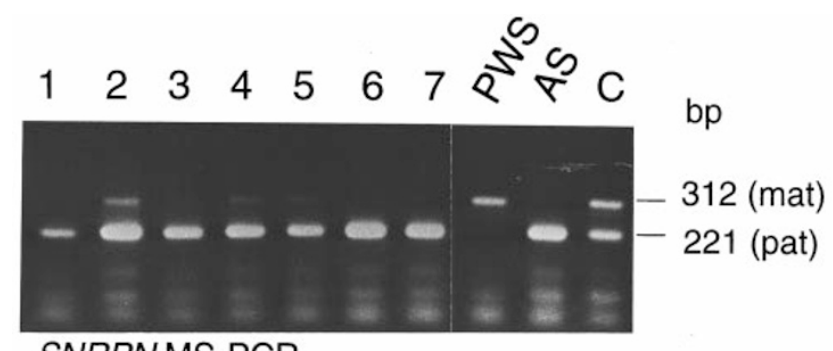

SNRPN MS-PCR

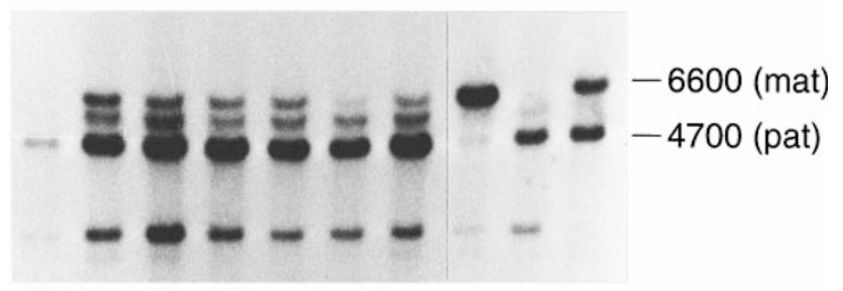

PW71, Hind III + Hpa II

Figure 3 Methylation analysis. Genomic DNA samples were analysed with the methylation-specific PCR (MS-PCR, upper part of Figure) and Southern blot hybridisation with PW71B (lower part of Figure). 1-7, patients, PWS, PWS control (typical deletion); $A S, A S$ control (typical deletion); $C$, normal control. Note weak maternal MS-PCR bands in patients 2, 4 and 5. Patients 1 and 3 had a very weak maternal band, which does not reproduce in this photograph
In contrast to previous genotype-phenotype studies in AS, however, our patients were not ascertained on the basis of clinical features suggestive of AS, but because of early onset of obesity, muscular hypotonia and mental retardation. These features do not belong to the 'classic' spectrum of features in AS, but could be suggestive of PWS. Since methylation analysis detects PWS and AS in the same test, a molecular diagnosis of AS was made, although initially PWS was suspected in the patients. Interestingly, patients $1-5$ had a weak maternal band in the SNRPN test (Figure 3). Patients 6 and 7 had a typical methylation pattern and are more retarded than the other five patients. On the other hand, in a recent genotype-phenotype study, Moncla et $a l^{5}$ described non-deleted AS patients who had a behavioural phenotype similar to PWS consisting of hyperphagia and an increase in weight. The phenotypic overlap of patients in this and our study may suggest that the atypical phenotype here represents the very end of a continuous phenotypic spectrum of AS patients.

Notably, all the patients described here have an imprinting defect. This classification is based on the finding of an abnormal methylation pattern in the presence of normal chromosomes of biparental origin. ${ }^{7,19}$ All cases are sporadic, and we have not found any evidence for an imprinting centre microdeletion. The mild phenotype may be due to an incomplete imprinting defect or to cellular mosaicism. As we cannot test the methylation and expression pattern of the $A S$ gene(s) in brain, it is possible that the imprinting defect does not extend from the imprinting centre to these genes in each cell. Alternatively, some of the patients, especially those presenting with a weak maternal SNRPN band, may be somatic mosaics in which cells with a normal imprint and cells with an imprinting defect coexist. Mosaicism may result from a 
postzygotic error of imprint maintenance or from the postzygotic correction of an imprint incorrectly set in the parental germline. At present, it is not possible to distinguish between these possibilities.

Attempts to explain the phenotype in the patients described here are hampered by incomplete knowledge about the genes involved in AS and PWS. At present it is not clear whether $U B E 3 A$ is the only $A S$ gene, or whether the $G A B R B 3$ gene or other genes contribute to the phenotype, at least in patients with a deletion. ${ }^{20}$ Furthermore, the imprinting status of $G A B R B 3$ is a matter of debate. ${ }^{21}$ Last, but not least, the etiology of obesity in PWS is still unknown. In PWS obesity usually starts in early childhood, whereas our patients with AS were already overweight in their first year of life. This observation suggests that different genes might play a role in the etiology of obesity in the two conditions. On the other hand, the presence of muscular hypotonia in both groups of patients may point to the involvement of the same gene(s). Nevertheless, it is difficult to explain the presence of these features in patients with PWS, who lack a paternal contribution in 15q11-q13, and in AS patients with an imprinting defect and uniparental disomy, who have a double dosage of paternally expressed genes.

In view of the fact that major features of AS are absent in the seven patients reported here, it raises the question whether these patients should be classified as AS solely on the basis of their methylation pattern. From a molecular point of view, the answer is yes, and the patients extend the currently known phenotypic spectrum of AS. From a clinical point of view, however, the answer is less clear. These patients lack several of the most characteristic features of AS. However, we think, that AS patients of different molecular classes share important features like mental retardation and speech problems. Therefore, such children may benefit from joining an AS support group.

In conclusion, we have identified a group of patients with a chromosome 15 imprinting defect who have atypical AS. The absence of speech was hitherto regarded as an almost mandatory feature of AS. Following current diagnostic criteria, an experienced clinician would not have made the diagnosis of AS or PWS in these patients. Our accidental finding of these patients reinforces the idea of performing a methylation test in all children presenting with obesity, muscular hypotonia and mental retardation. Knowing the phenotype of the patients described here may also help to recognise this form of AS more often.

\section{Acknowledgements}

We thank Dr R Fahsold, Dresden, for molecular studies of patient 3, Dr M Hagen, Dr A Petrick and Dr Hasselmann for referring patients 2, 3 and 6; Dr K Buiting, C Färber and Professor E Passarge for helpful discussions, and the Deutsche Forschungsgemeinschaft for financial support.

\section{References}

1 Angelman H: Puppet children: A report of three cases. Dev Med Child Neurol 1965; 7: 681-688.

2 Clayton-Smith J: Clinical research on Angelman syndrome in the United Kingdom: Observations on 82 affected individuals. Am J Med Genet 1993; 46: 12-15.

3 Williams CA, Angelman H, Clayton-Smith J et al: Angelman syndrome: consensus for diagnostic criteria. Am J Med Genet 1995; 56: 237-238.

4 ASHG/ACMG: Diagnostic testing for Prader-Willi and Angelman syndromes: Report of the ASHG/ACMG test and technology transfer committee. Am J Hum Genet 1996; 66: 77-80.

5 Moncla A, Malzac P, Voelckel MA et al: Phenotypegenotype correlation in 20 deletion and 20 non-deletion Angelman syndrome patients. Eur J Hum Genet 1999; 7: 131-139.

6 Buiting K, Dittrich B, Groß S et al: Sporadic imprinting defects in Prader-Willi syndrome and Angelman syndrome: Implications for imprint switch models, genetic counseling and prenatal diagnosis. Am J Hum Genet 1998; 63: $170-180$.

7 Horsthemke B, Dittrich B, Buiting K: Imprinting mutations on human chromosome 15. Hum Mutat 1997; 10: 329-337.

8 Bürger J, Kunze J, Sperling K, Reis A: Phenotypic differences in Angelman syndrome patients: Imprinting mutations show less frequently microcephaly and hypopigmentation than deletions. Am J Med Genet 1996; 66: 221-226.

9 Bottani A, Robinson WP, DeLoizier-Blanchet CD et al: Angelman syndrome due to paternal uniparental disomy of chromosome 15: A milder phenotype? Am J Med Genet 1994; 51: 35-40.

10 Gillessen-Kaesbach G, Albrecht B, Passarge E, Horsthemke B: Further patient with Angelman syndrome due to paternal disomy of chromosome 15 and milder phenotype. Am J Med Genet 1995; 56: 328-329.

11 Fridman C, Varela MC, Nicholls RD, Koiffmann CP Unusual clinical features in an Angelman syndrome patient with uniparental disomy due to a translocation 15q15q. Clin Genet 1998; 54: 303-308.

12 Smith A, Robson L, Buchholz B: Normal growth in Angelman syndrome due to paternal UPD. Clin Genet 1998; 53: 223-225.

13 Seabright M: A rapid banding technique for human chromosomes. Lancet 1971; 2: 971-972.

14 Lichter P, Chang CJ, Call K et al: High resolution mapping of human chromosome 11 by in situ hybridization with cosmid clones. Science 1990; 247: 64-67.

15 Dittrich B, Buiting B, Horsthemke B: PW71 methylation test for Prader-Willi and Angelman syndrome. Am J Hum Genet 1996; 61: 196-197. 
16 Zeschnigk M, Lich C, Buiting K, Doerfler W, Horsthemke $\mathrm{B}$ : A single-tube PCR test for the diagnosis of Angelman and Prader-Willi syndrome based on allelic methylation differences at the SNRPN locus. Eur J Hum Genet 1997; 5: 94-98.

17 Schumacher A, Buiting K, Zeschnigk M, Doerfler W, Horsthemke B: Methylation analysis of the PWS/AS region does not support an enhancer-competition model. Nat Genet 1998; 19: 324-325.

18 Minassian BA, DeLorey TM, Olsen RW et al: Angelman syndrome: Correlations between epilepsy phenotypes and genotypes. Ann Neurol 1998; 43: 485-493.
19 Nicholls RD, Saitoh S, Horsthemke B: Imprinting in Prader-Willi and Angelman syndromes. Trends Genet 1998; 14: 194-199.

20 DeLorey TM, Handforth A, Anagnostaras SG et al: Mice lacking the beta3 subunit of the GABAA receptor have the epilepsy phenotype and many of the behavioral characteristics of Angelman syndrome. J Neurosci 1998; 18: $8505-8514$.

21 Meguro M, Mitsuya K, Sui $\mathrm{H}$ et al: Evidence for uniparental, paternal expression of the human $\mathrm{GABA}_{\mathrm{A}}$ receptor subunit genes, using microcell-mediated chromosome transfer. Hum Mol Genet 1997; 12: 2127-2133. 